\title{
Depósitos Unitários de Calda de Pulverização com e sem SuRfatante em Plantas de Salvinia molesta ${ }^{1}$
}

\author{
Spray Solution Deposit on Salvinia molesta With and Without Surfactant
}

\begin{abstract}
NEGRISOLI, E. ${ }^{2}$, TOFOLI, G.R. ${ }^{3}$, VELINI, E.D. ${ }^{4}$, MARTINS, D. ${ }^{4}$ e PALLADINI, L.A. ${ }^{5}$
RESUMO - Uma das opções para o manejo de Salvina molesta é o controle químico; contudo, a presença de grande quantidade de pêlos na epiderme foliar reduz a molhabilidade das folhas, o que pode afetar a eficiência dos herbicidas. O objetivo deste trabalho foi o de avaliar a deposição do corante azul FDC-1, no qual se simulou a aplicação de herbicidas em plantas dessa espécie, com e sem a mistura de um surfatante. Os tratamentos foram as concentrações de 0 e $5 \%$ do surfatante Aterbane (espalhante adesionante), utilizado na elaboração da calda de pulverização. O delineamento experimental foi o inteiramente casualizado, com 150 repetições. A aplicação foi realizada com um pulverizador estacionário à pressão de 2,0 bar, com consumo de calda de $180 \mathrm{~L} \mathrm{ha}^{-1}$. Foram utilizados bicos de jato plano, tipo XR 110.02 . Foram ajustadas curvas de regressão entre os depósitos individuais em cada planta ( $\mu \mathrm{L}$ calda/ planta) e as freqüências acumuladas. Utilizou-se o modelo de Gompertz, e os valores de $\mathrm{R}^{2}$ foram de 0,99 e 0,97 com e sem o espalhante, respectivamente. Em termos médios, a adição de Aterbane reduziu em 1,13\% os depósitos do FDC-1. No entanto, o espalhante melhorou em 76, 41 e 29\% a deposição em 1, 5 e 10\% da população com menores depósitos do corante.
\end{abstract}

Palavras-chave: salvínia, planta aquática, surfatante, deposição, gotas.

ABSTRACT - One of the options for managing Salvinia molesta is chemical control; however, the presence of a large amount of stiff, tiny water resistant hairs on their leaf surface above reduces leaf wettability and herbicide efficiency. The objective of this study was to evaluate the deposition of blue coloring FDC-1, by simulating the application of herbicide solutions on this aquatic fern, with and without a surfactant mixture. The treatments were concentrations of Aterbane at 0 and $5 \%$, used in a spray solution preparation. The experimental treatment was set up on a randomized design with 150 replications. Application was performed with a stationary spray with pressure at 2.0 bar and consumption of $180 \mathrm{~L} \mathrm{ha}^{-1}$ of spray solution. Nozzles type XR 110.02 were used and regression curves were adapted among the individual deposits in each plant ( $\mathrm{mL}$ spray solution/ plant) and to the accumulated frequencies. The model of Gompertz was used and the $R^{2}$ values were 0.99 and 0.97, with and without Aterbane, respectively. In average, addition of Aterbane spray solution reduced deposits of the FDC-1 in 1.13\%. However, Atherbane improved the deposition of $76 \%, 41 \%$ and $29 \%$ in $1 \%, 5 \%$ and $10 \%$, of the population, respectively, with less deposit of the coloring.

Key words: weed, aquatic plant, surfactant, deposition, drops.

\section{INTRODUÇÃO}

As plantas aquáticas são parte integrante dos ecossistemas aquáticos e desempenham funções vitais na manutenção destes, entre as quais a oxigenação da água, o refúgio para organismos vertebrados e invertebrados, a proteção contra a ação erosiva e a remoção de nutrientes da água, além de serem fonte de alimento para diversos organismos (IBAMA, 1998).

Recebido para publicação em 21/11/2001e na forma revisada em 20/12/2001.

2 Eng.-Agr., M.S., aluno de Pós-graduação em nível de Doutorado, curso de Agricultura, FCA-UNESP, 18603-970 BotucatuSP, <ednegri@fca.unesp.br>; ${ }^{3}$ Eng.-Agr., M.S., aluno de Pós-Graduação em nível de Doutorado, curso de Proteção de Plantas, FCA-UNESP, Botucatu-SP. ${ }^{4}$ Professores Drs. Docentes do Departamento de Produção Vegetal da FCA-UNESP, Botucatu-SP. Pesquisador da EPAGRI, Caçador-SC. ${ }^{5}$ Pesquisador Dr. da Epagri Caçador-SC, <palladini@ provinet.com.br>. 
A proliferação de plantas infestantes aquáticas em rios e lagos constitui-se em um sério e complexo problema, que tem repercussões não apenas em nível ambiental, mas atingem de igual modo esferas econômicas e sociais, como: pesca, recreação, navegação, retenção de lixo, problemas na geração de energia elétrica e refúgios a insetos.

A salvínia (Salvinia molesta) está listada entre as piores espécies de plantas de hábito flutuante das mais diversas coleções d'água do planeta, juntamente com Pistia stratiotes e Euchhornia crassipes, segundo Lorenzi (1992). É uma planta extremamente agressiva, por apresentar rápida multiplicação e fácil reprodução. Uma das opções para o manejo dessa espécie é o controle químico, porém a presença na epiderme foliar de grande quantidade de pêlos dificulta a coalescência das gotas de pulverizações, o que reduz a molhabilidade das folhas e provavelmente a eficiência dos herbicidas.

Martins et al. (1999) estudaram o controle químico de Salvinia molesta, em condições de caixa d'água, e observaram que os herbicidas 2,4 D e glyphosate não foram eficientes em seu controle. Esse fato, segundo os pesquisadores, poderia estar talvez relacionado à grande quantidade de pêlos em sua epiderme. Devese ressaltar que essa estratégia da planta auxilia-a não submergir e seria também uma forma de não absorver herbicidas, mesmo os aplicados sobre suas folhas ou diretamente na água.

Os controles insatisfatórios observados no campo provavelmente podem ser atribuídos à falta de um contato íntimo do produto com a planta. Não há informações na literatura que avaliem a deposição pontual de herbicidas em plantas aquáticas, justificando-se a realização deste estudo, principalmente em plantas com as características descritas. Assim, o objetivo deste experimento foi o de avaliar a deposição de caldas de pulverização, com e sem a adição de surfatante, sobre folhas de plantas de salvínia.

\section{MATERIAL E MÉTODOS}

O experimento foi conduzido no Núcleo de Pesquisas Avançadas em Matologia (NUPAM), pertencente ao Departamento de Produção
Vegetal da Faculdade de Ciências Agronômicas/ UNESP - campus de Botucatu-SP.

As plantas de salvinia foram coletadas nos tanques de piscicultura da CESP, localizados em Barra Bonita-SP, transportadas para Botucatu e acondicionadas em caixas d'água no NUPAM. Para este estudo foram selecionadas as plantas de salvínia que continham quatro folhas desenvolvidas e duas em início de desenvolvimento, o que permitiu instalar o estudo com as plantas bem uniformes.

As plantas selecionadas foram colocadas em 40 vasos de plástico com capacidade para $200 \mathrm{~mL}$. Os vasos foram colocados de forma que ficassem posicionados por toda a extensão da área útil da barra de aplicação.

Como tratamentos utilizaram-se as concentrações de 0 e $5 \%$ do espalhante adesionante Aterbane. A calda de pulverização foi preparada com $3 \mathrm{~g} \mathrm{~L}^{-1}$ do corante azul FDC- 1 . Deve-se ressaltar que este corante não influi nas características físicas da calda, como a tensão superficial das gotas pulverizadas. No estudo, o corante foi utilizado como um traçante para simulação de uma aplicação de herbicida, conforme metodologia descrita por Palladini (2000).

A aplicação foi feita com um pulverizador estacionário a pressão constante de $\mathrm{CO}_{2}$ a 2,0 bar, com um consumo de calda de $180 \mathrm{~L} \mathrm{ha}^{-1}$, sobre 40 vasos contendo as plantas de salvínia. Na aplicação foram utilizados quatro bicos de jato plano, espaçados de 0,5 m entre si, tipo XR 110.02, acoplados a uma barra de $2,0 \mathrm{~m}$.

Após a aplicação foram coletadas 150 plantas de salvínia para cada tratamento, com e sem Aterbane. Cada planta correspondeu a uma repetição. O delineamento experimental utilizado foi o inteiramente casualizado. Essas plantas foram imediatamente lavadas com 25 mL de água deionizada, para retirar o corante que tinha sido depositado durante a sua aplicação, o que originou as amostras enviadas para análise.

As amostras foram quantificadas em espectrofotômetro (GBC Cintra 40), sendo determinadas as concentrações do corante depositado sobre as plantas no comprimento de onda de $630 \mathrm{~nm}$. Foram obtidos os dados 
de absorbância, que foram transformados em dados de volume ( $\mu \mathrm{L}$ de calda/planta), através da expressão matemática $\mathrm{C}_{1} \cdot \mathrm{V}_{1}=\mathrm{C}_{2} \cdot \mathrm{V}_{2}$, em que:

$\mathrm{C}_{1}$ - concentração da calda no momento da aplicação;

$\mathrm{V}_{1}$ - quantidade em $\mathrm{mL}$ que depositou nas plantas de salvínia;

$\mathrm{C}_{2}$ - leitura de concentração da amostra; e

$\mathrm{V}_{2}-25 \mathrm{~mL}$ de água deionizada (lavagem).

Os dados dos depósitos em $\mu \mathrm{L}$ de calda/ planta foram ajustados pelo modelo de Gompertz, $\mathrm{y}=\mathrm{e}^{* *}\left(\mathrm{a}-\mathrm{e}^{* *}\left(-\mathrm{b}-\mathrm{c}^{*} \mathrm{x}\right)\right)$, em que:

$\mathrm{y}=$ freqüência acumulada $\operatorname{dos} \operatorname{dados}(\mu \mathrm{L}$ de calda/planta);

$\mathrm{x}=$ depósitos em $\mu \mathrm{L}$ de calda/planta;

$\mathrm{a}=$ valor estimado pelo modelo;

$\mathrm{b}=$ valor estimado pelo modelo; $\mathrm{e}$

$\mathrm{c}=$ valor estimado pelo modelo.

O modelo de Gompertz apresenta-se assimétrico; conseqüentemente, a primeira derivada desse modelo mostrou-se também assimétrica e representa as curvas de freqüência não-acumulada ou densidade de probabilidade dos dados de depósito em $\mu \mathrm{L}$ de calda/planta. Foram calculados os valores de $\mathrm{R}^{2}$ (coeficiente de determinação) para cada equação obtida (com e sem Aterbane).

\section{RESULTADOS E DISCUSSÃO}

Na Figura 1 estão apresentados os resultados que correspondem às freqüências acumuladas em função da deposição representada em $\mu \mathrm{L}$ de calda/planta dos tratamentos com e sem Aterbane. O gráfico foi elaborado utilizando-se os dados originais, sem ajuste. Devese ressaltar que as curvas menos inclinadas indicam maior dispersão dos dados e maiores variações dos depósitos unitários.

Observa-se, para a curva de deposição unitária do tratamento sem Aterbane, que houve pequena variação (menor inclinação) em relação aos dados do tratamento com Aterbane. Desse modo, é provável que os valores de deposição do tratamento sem Aterbane estejam mais dispersos, ou seja, a variação entre eles é maior.

Os dados originais foram submetidos aos ajustes feitos pelo modelo de Gompertz. A partir desses ajustes, foram confeccionadas as curvas de freqüência acumulada, em que foram acertados os dados de depósitos do traçante (corante FDC-1) em $\mu 1$ de calda/planta, para a aplicação com e sem Aterbane (Figura 2).

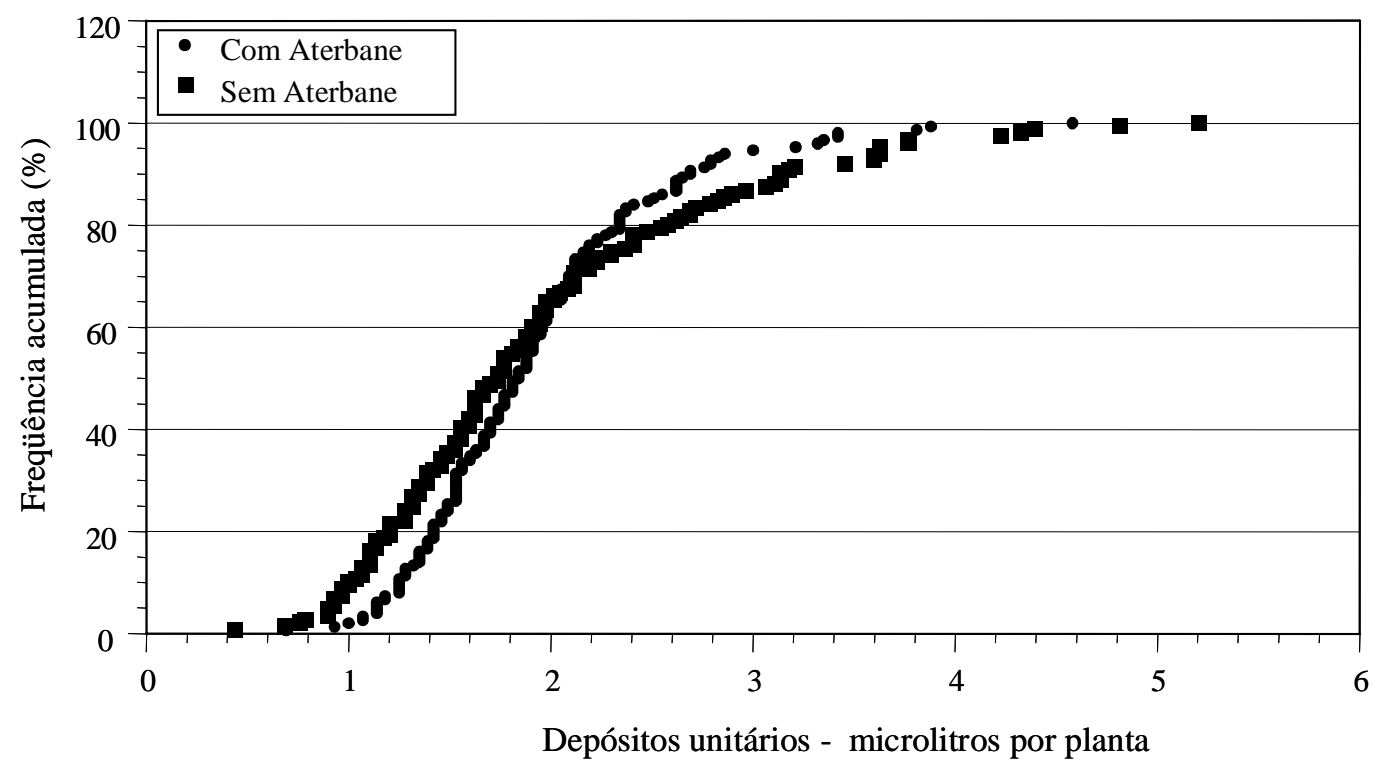

Figura 1 - Freqüências acumuladas em função da deposição representada em $\mu \mathrm{L}$ de calda/planta. 


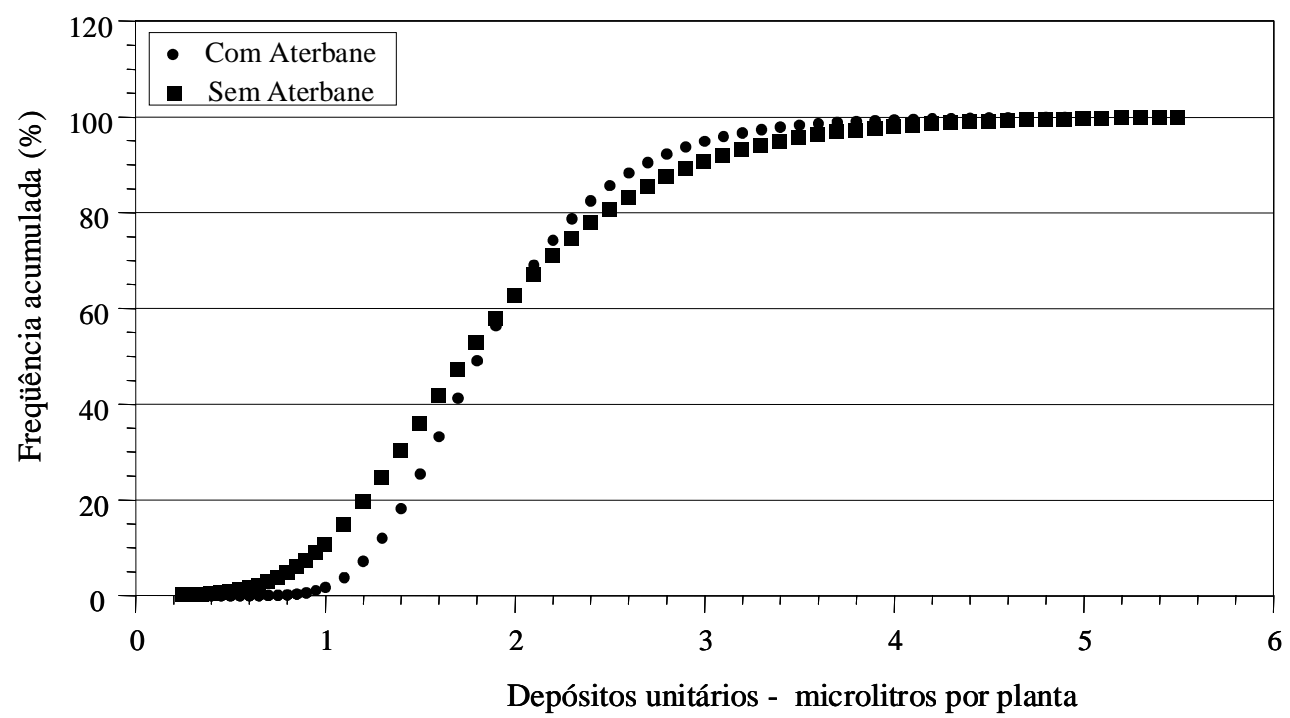

Figura 2 - Freqüências acumuladas em função dos depósitos do corante FDC-1 em $\mu \mathrm{L}$ de calda/planta para a aplicação com e sem Aterbane.

Os resultados da análise de regressão mostraram que os coeficientes de determinação para os depósitos em $\mu \mathrm{L}$ de calda/planta nos dois tratamentos (com e sem Aterbane) foram de 0,99 e 0,97, respectivamente.

Na Figura 3 estão apresentados os resultados da derivada primeira do modelo de Gompertz, que representa a densidade de probabilidade, dos depósitos do corante FDC$1 \mathrm{em} \mu \mathrm{L}$ de calda/planta. Analisando as curvas desse gráfico, observa-se que para os dados de freqüência não-acumulada, comparada pela adição ou não de Aterbane, a curva da aplicação com Aterbane apresenta-se mais íngreme em relação à aplicação sem Aterbane. Portanto, a calda de aplicação, quando adicionada com o surfatante Aterbane, conferiu maior uniformidade de depósitos.

Um outro resultado importante, relacionado com a média, refere-se à aplicação sem Aterbane, no qual as médias dos depósitos do corante foram superiores. Contudo, esse fator não deve ser considerado, pois a média avalia quantitativamente a pulverização, e o mais importante seria a análise qualitativa da pulverização, ou seja, a dispersão dos dados de depósitos. Assim, fica evidente a melhora na deposição da aplicação sobre as plantas de salvínia, causada pela adição do surfatante
Aterbane à calda de pulverização. Tofoli (2001), estudando o efeito do tamanho do alvo e das condições operacionais sobre a uniformidade de deposição de pulverizações em pré-emergência, verificou que a média não foi um bom parâmetro para avaliar esse tipo de estudo.

Na Figura 4 está representada, em porcentagem, a relação dos depósitos (com e sem Aterbane) em função da freqüência acumulada. Nota-se que, em termos médios, a adição de Aterbane reduziu em 1,13\% os depósitos do corante. No entanto, o espalhante Aterbane melhorou em 76, 41 e 29\% a deposição em 1, 5 e $10 \%$ da população com menores depósitos de corante.

Os 1, 5 e 10\% da população, em que menos ocorreram depósitos, podem ser responsáveis pelo insucesso do controle em plantas de salvínia. Martins et al. (1999) verificaram que os herbicidas imazapyr, 2,4 D e glyphosate não foram eficientes no controle de S. molesta, em todas as doses testadas na ocasião.

As irregularidades dos depósitos podem levar à necessidade de aumentos na dose aplicada, que podem sobrelevar em várias vezes os incrementos de doses necessárias para compensar as perdas. Apesar disso, estudos que procuraram estabelecer a dispersão de depósitos no solo ou em populações de plantas 


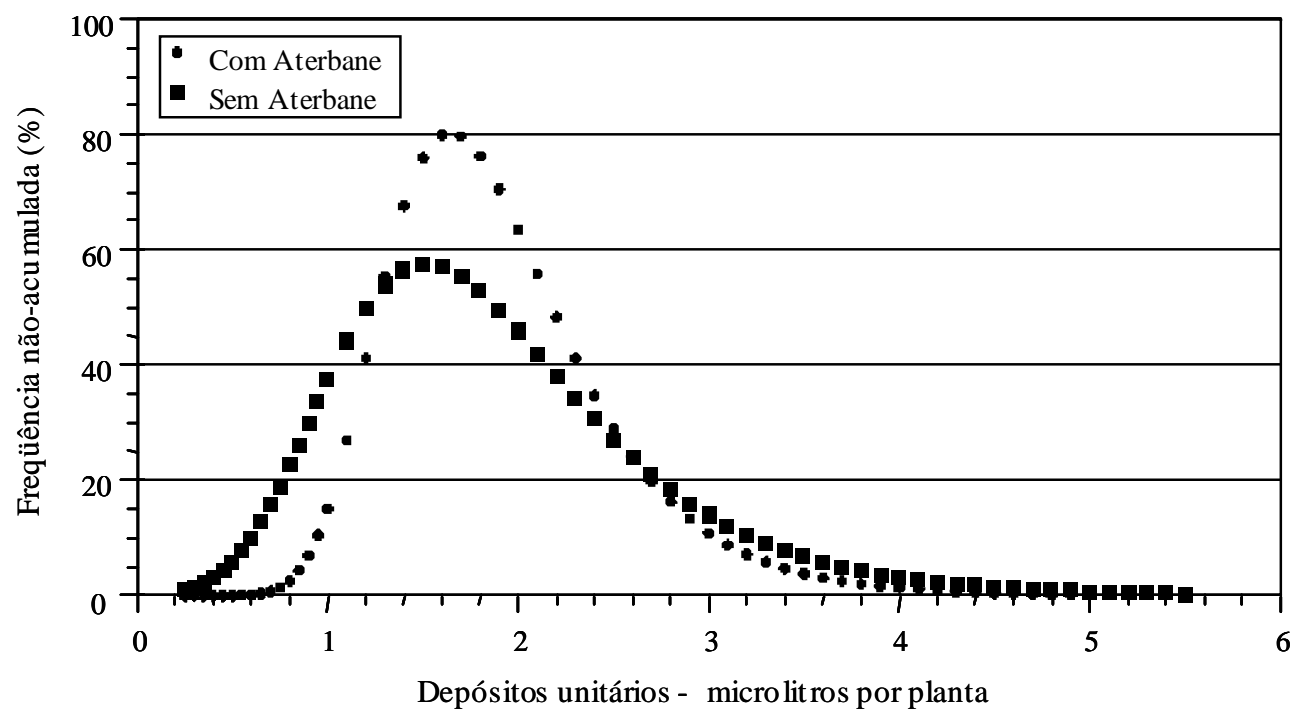

Figura 3 - Densidade de probabilidade em $\mu \mathrm{L}$ de calda/planta dos depósitos do corante nos tratamentos com e sem aterbane.

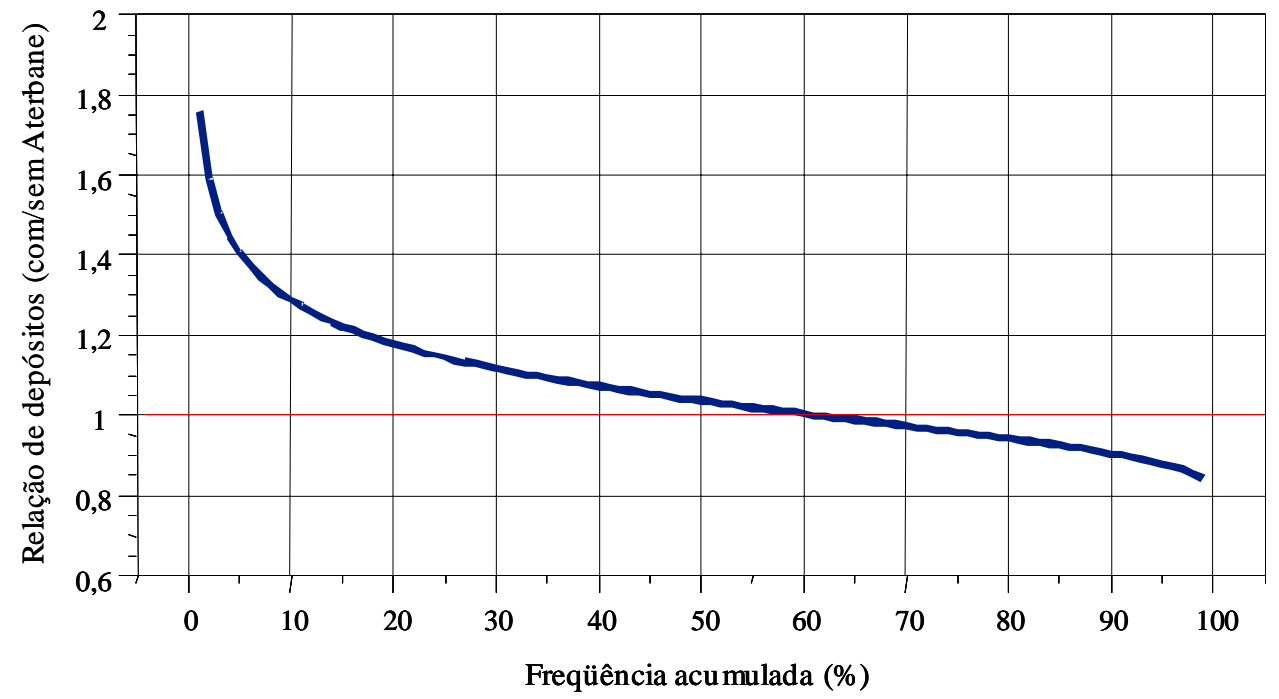

Figura 4 - Relação entre os depósitos de corante para as pulverizações com e sem Aterbane. Dados ajustados.

daninhas e/ou aquáticas são praticamente inexistentes. Exceções são os trabalhos de Souza et al. (2000a), em que foram estudados depósitos unitários de calda de pulverização em plantas daninhas da cultura da soja. Souza (2000b) analisou também os depósitos unitários de calda de pulverização em reboleiras de plantas daninhas em aplicações de manejo. Já Tofoli (2001) analisou o efeito do tamanho do alvo e das condições operacionais sobre a uniformidade de deposição de pulverizações em pré-emergência.

\section{LITERATURA CITADA}

INSTITUTO BRASILEIRO DE MEIO AMBIENTE E DOS RECURSOS NATURAIS RENOVÁVEIS - IBAMA. In: WORKSHOP CONTROLE DE PLANTAS AQUÁTICAS, 1988, Brasília. Resumos... Brasília: IBAMA, 1998. p. 1-3.

LORENZI, H. Plantas daninhas do Brasil. Nova Odessa: 1992. $40 \mathrm{p}$.

MARTINS, D. et al. Controle químico de plantas daninhas aquáticas em condições controladas - caixa d'água. Planta Daninha, v. 17, n. 2, p. 289-96, 1999. 
PALLADINI, L. A. Metodologia para avaliação da deposição em pulverizações. Botucatu, Universidade Estadual Paulista, 2000. 111 p. Tese (Doutorado em Agronomia/Proteção de Plantas) - Universidade Estadual Paulista, 2000

SOUZA, R. T.; MACIEL, C. D. G.; VELINI, E. D. Avaliação dos depósitos unitários de calda de pulverização em plantas daninhas da cultura da soja. In: CONGRESSO BRASILEIRO DA CIÊNCIA DAS PLANTAS DANINHAS, 2000 Foz do Iguaçu. Anais de Resumo... Foz do Iguaçu: 2000a. p. 473.
SOUZA, R. T. et al. Depósitos unitários de calda de pulverização em reboleiras de plantas daninhas em aplicações de manejo. In: CONGRESSO BRASILEIRO DA CIÊNCIA DAS PLANTAS DANINHAS, 22, 2000, Foz do Iguaçu. Resumos... Foz do Iguaçu: 2000b. p. 474.

TOFOLI, G. R. Efeito do tamanho do alvo e condições operacionais sobre a uniformidade de deposição de pulverizações em pré-emergência. Botucatu: Universidade Estadual Paulista, 2001. 62 p. Dissertação (Mestrado em Agronomia/Proteção de Plantas) - Universidade Estadual Paulista, 2001. 